\title{
SEMI MODUL POLINOMIAL FUZZY ATAS ALJABAR MAX-PLUS FUZZY
}

\author{
Ari Wardayani dan Suroto \\ Prodi Matematika, Jurusan MIPA, Fakultas Sains dan Teknik \\ Universitas Jenderal Soedirman \\ (email : ariwardayani@yahoo.co.id, suroto_80@yahoo.com)
}

\begin{abstract}
ABSTRAK. Pada makalah ini dibahas mengenai perluasan aljabar max-plus fuzzy pada polinomial dengan koefisien bilangan fuzzy. Selanjutnya, dibuktikan polinomial fuzzy tersebut merupakan semi modul atas aljabar max-plus fuzzy.

Kata kunci : aljabar max-plus fuzzy, polinomial fuzzy, semi modul
\end{abstract}

\begin{abstract}
In this paper, we discuss the extension of fuzzy max-plus algebra on polynomial with coefficient in fuzzy number. We also proof that fuzzy polynomial is semi modul over fuzzy max-plus algebra.
\end{abstract}

Key word : fuzzy max-plus algebra, fuzzy polynomial, semi modul

\section{PENDAHULUAN}

Aljabar max-plus merupakan struktur aljabar $\mathbb{R}_{\max }=\mathbb{R} \cup\{-\infty\}$ yang disertai dengan dua operasi biner yakni maksimum dan penjumlahan. Struktur aljabar $\mathbb{R}_{\max }$ yang disertai dengan operasi biner maksimum sebagai operasi $\oplus$ dan operasi penjumlahan sebagai operasi $\otimes$ adalah semi lapangan komutatif idempoten (Bacelli, 2001). Pada dasarnya, himpunan yang dibicarakan dalam pembahasan konsep aljabar max-plus lebih terpusat pada himpunan bilangan real $\mathbb{R}$.

Namun, dalam perkembangan selanjutnya aljabar max-plus dapat diperluas himpunan pembicaraannya menjadi himpunan bilangan fuzzy (Rudhito, 2006). Bilangan fuzzy adalah himpunan fuzzy dalam semesta $\mathbb{R}$ yang memenuhi sifat normal, mempunyai support terbatas, setiap $\alpha$-cutnya merupakan selang 
tertutup, dan konveks. Untuk selanjutnya himpunan bilangan fuzzy dilambangkan dengan $\mathcal{R}$. Operasi maksimum dan penjumlahan pada $\mathcal{R}$ dapat didefinisikan dengan menggunakan prinsip perluasan atau dengan $\alpha$-cut pada himpunan fuzzy (Zimmermann, 1991).

Teorema dekomposisi merupakan landasan kerja pemanfaatan prinsip perluasan dan $\alpha$-cut pada himpunan fuzzy yang dapat digunakan untuk menentukan operasi aritmatika pada bilangan fuzzy (Susilo, 2006). Untuk selanjutnya aljabar max-plus fuzzy dinotasikan dengan $\mathcal{R}_{\max }$, dengan $\mathfrak{R}$ adalah himpunan bilangan fuzzy. Pada tahun 2007, Rudhito membuktikan semi modul bilangan fuzzy atas aljabar max-plus fuzzy dan perluasannya pada matriks bilangan fuzzy. Pada tulisan ini, akan dibuktikan bahwa himpunan polinomial fuzzy merupakan semi modul atas semi ring aljabar max-plus fuzzy.

\section{SEMI MODUL POLINOMIAL FUZZY}

Semi ring $\mathcal{K}$ merupakan suatu himpunan tak kosong yang disertai dengan dua operasi biner $\bigoplus$ dan $\bigotimes$ yang memenuhi $(\mathcal{K}, \bigoplus)$ semi grup komutatif dengan elemen nol $\varepsilon,(\mathscr{K}, \bigotimes)$ semi grup dengan elemen satuan $e$, elemen nol $\varepsilon$ merupakan elemen penyerap terhadap operasi $\otimes$, dan $\otimes$ distributif terhadap $\oplus$. Suatu semi ring dikatakan idempoten jika operasi $\oplus$ bersifat idempoten dan dikatakan komutatif jika operasi $\otimes$ bersifat komutatif.

Semi modul $\mathscr{N}$ atas semi ring $\mathscr{K}$ adalah himpunan tak kosong yang disertai operasi internal $\bigoplus$ dengan elemen nol $\varepsilon$, dan operasi eksternal yang didefinisikan pada $\mathcal{K} x \mathscr{K}$ dengan hasilnya pada $\mathscr{T}$ yang memenuhi operasi $\bigoplus$ bersifat assosiatif, komutatif dan untuk setiap $\alpha, \beta \in \mathscr{K}$ dan $x, y \in$ Or berlaku $\quad \alpha(x \oplus$ $y)=\alpha x \oplus \alpha y,(\alpha \oplus \beta) x=\alpha x \oplus \beta x, \alpha(\beta x)=(\alpha \beta) x$, ex $=x$ dan $\quad \varepsilon x=\varepsilon$

Misalkan dibentuk suatu himpunan yang beranggotakan polinomialpolinomial dengan indeterminate $\gamma$ dan koefisiennya bilangan fuzzy

$$
\left\{p \mid p=\bigoplus_{i=0}^{n} \tilde{a}_{i} \gamma^{i}, \tilde{a}_{i} \in \mathcal{R}_{\max }\right\}
$$


untuk suatu $n \in \mathbb{N}$. Untuk selanjutnya, himpunan ini di notasikan dengan $\mathscr{R}_{\max }[\gamma]$ dan dinamakan himpunan polinomial fuzzy. Misalkan $p$ dan $q$ elemen pada $\mathcal{R}_{\max }[\gamma]$ dengan $p=\bigoplus_{i=0}^{n} \tilde{a}_{i} \gamma^{i}$ untuk $\tilde{a}_{i} \in \mathcal{R}_{\max }, q=\bigoplus_{i=0}^{m} \tilde{b}_{i} \gamma^{i}$ untuk $\tilde{b}_{i} \in \mathcal{R}_{\max }$. Elemen $p$ dan $q$ dikatakan sama jika $m=n$ dan $\tilde{a}_{i}=\tilde{b}_{i}$.

Untuk menyelidiki sifat-sifat yang terdapat pada struktur $\mathcal{R}_{\max }[\gamma]$ terlebih dahulu didefinisikan dua operasi pada $\mathcal{R}_{\max }[\gamma]$ yakni operasi internal $\oplus$ sebagai operasi penjumlahan komponen demi komponen pada polinomial, dan operasi eksternal pergandaan dengan skalar pada $\mathscr{R}_{\max }$.

Untuk setiap $p, p^{\prime}, q, q^{\prime} \in \Re_{\max }[\gamma]$ dengan $p=p^{\prime}$ dan $q=q^{\prime}$. Misalkan $p=\bigoplus_{i=0}^{n} \tilde{a}_{i} \gamma^{i}$ untuk $\tilde{a}_{i} \in \mathcal{R}_{\max }$, dan $p^{\prime}=\bigoplus_{i=0}^{m} \tilde{b}_{i} \gamma^{i}$ untuk $\tilde{b}_{i} \in \mathcal{R}_{\max }$. Karena $p=p$, maka $\tilde{a}_{i}=\tilde{b}_{i}$ diperoleh $\tilde{a}_{i \alpha}=\tilde{b}_{i \alpha}$ untuk setiap $\alpha \in[0,1], i=1,2, \ldots, n$ dan $n=m$. Secara analog, misalkan $q=\bigoplus_{i=0}^{k} \quad \tilde{c}_{i} \gamma^{i}$ untuk $\tilde{c}_{i} \in \mathscr{R}_{\max }$, dan $q^{\prime}=\bigoplus_{i=0}^{l} \tilde{d}_{-} \gamma^{i}$ untuk $\tilde{d}_{i} \in \mathcal{R}_{\max }$. Karena $q=q$ ', maka $\tilde{c}_{i}=\tilde{d}_{i}$ diperoleh $\tilde{c}_{i \alpha}=\tilde{d}_{i \alpha}$ untuk setiap $\alpha \in[0,1], i$ $=1,2, \ldots, k$ dan $k=l$. Tanpa mengurangi keumuman, misalkan $n \geq k$ dan $m \geq l$. Dengan demikian,

$$
\begin{aligned}
& p \oplus q=\left(\bigoplus_{i=0}^{\text {雬 }} \tilde{a}_{i} \gamma^{i}\right) \oplus\left(\bigoplus_{i=0}^{k} \tilde{c}_{i} \gamma^{i}\right)=\bigoplus_{i=0}^{n} \tilde{t}_{i} \gamma^{i}, \text { dengan } \tilde{t}_{i}=\tilde{a}_{i} \widetilde{\oplus} \tilde{c}_{i} \\
& p^{\prime} \oplus q^{\prime}=\left(\bigoplus_{i=0}^{m} \quad \tilde{b}_{i} \gamma^{i}\right) \bigoplus\left(\bigoplus_{i=0}^{l} \tilde{d}_{i} \gamma^{i}\right)=\bigoplus_{i=0}^{n} \tilde{s}_{i} \gamma^{i}, \text { dengan } \tilde{s}_{i}=\tilde{b}_{i} \widetilde{\oplus} \tilde{d}_{i}
\end{aligned}
$$

Sementara itu, $\tilde{t}_{i}=\tilde{a}_{i} \widetilde{\oplus} \tilde{c}_{i}$ merupakan himpunan fuzzy yang $\alpha$-cutnya adalah interval $\left[\underline{a_{\alpha}} \bigoplus \underline{c_{\alpha}}, \overline{a_{\alpha}} \oplus \overline{c_{\alpha}}\right]$ untuk setiap $\alpha \in[0,1]$ dan $\left[\underline{a_{\alpha}} \bigoplus \underline{c_{\alpha}}, \overline{a_{\alpha}} \oplus \overline{c_{\alpha}}\right]=\left[\underline{a_{\alpha}}\right.$, $\left.\overline{a_{\alpha}}\right] \bigoplus\left[c_{\alpha}, \overline{c_{\alpha}}\right]$. Karena $\tilde{a}_{i}=\tilde{b}_{i}$, maka $\alpha$-cutnya sama, yakni $\tilde{a}_{i \alpha}=\tilde{b}_{i \alpha}$. Dari sini diperoleh $\left[\underline{a_{\alpha}}, \overline{a_{\alpha}}\right]=\left[\underline{b_{\alpha}}, \overline{b_{\alpha}}\right]$, sehingga $\underline{a_{\alpha}}=\underline{b_{\alpha}}, \overline{a_{\alpha}}=\overline{b_{\alpha}}$ dan analog untuk $\tilde{c}_{i}=\tilde{d}_{i}$. Disisi lain,

$$
\begin{aligned}
\tilde{t}_{i} & =\tilde{a}_{i} \widetilde{\oplus} \tilde{c}_{i}=\left[\underline{a_{\alpha}} \oplus \underline{c_{\alpha}}, \overline{a_{\alpha}} \oplus \overline{c_{\alpha}}\right]=\left[\underline{a_{\alpha}}, \overline{a_{\alpha}}\right] \oplus\left[\underline{c_{\alpha}}, \overline{c_{\alpha}}\right]=\left[\underline{b_{\alpha}}, \overline{b_{\alpha}}\right] \oplus\left[\underline{d_{\alpha}}, \overline{d_{\alpha}}\right] \\
& =\left[\underline{b_{\alpha}} \oplus \underline{d_{\alpha}}, \overline{b_{\alpha}} \oplus \overline{d_{\alpha}}\right]=\widetilde{b}_{i} \widetilde{\oplus} \widetilde{d}_{i}=\tilde{s}_{i}
\end{aligned}
$$


sehingga berlaku $p \oplus q=p^{\prime} \oplus q^{\prime}$. Dengan demikian, operasi internal $\oplus$ yang didefinisikan pada $\mathcal{R}_{\max }[\gamma]$ merupakan operasi yang terdefinisi dengan baik.

Selanjutnya, untuk setiap $p, p^{\prime} \in \mathcal{R}_{\max }[\gamma]$ dan $\widetilde{v}, \widetilde{w} \in \mathcal{R}_{\max }$ dengan $p=p^{\prime}$ dan $\widetilde{v}=\widetilde{w}$, diperoleh

$$
\begin{aligned}
& \widetilde{v} p=\widetilde{v}\left(\bigoplus_{i=0}^{n} \widetilde{a}_{i} \gamma^{i}\right)=\bigoplus_{i=0}^{n} \widetilde{v} \widetilde{a}_{i} \gamma^{i}=\bigoplus_{i=0}^{n} \widetilde{x}_{i} \gamma^{i} \quad \text { dengan } \widetilde{x}_{i}=\widetilde{v} \widetilde{a}_{i} \\
& \widetilde{w} p^{\prime}=\widetilde{w}\left(\bigoplus_{i=0}^{n} \widetilde{b}_{i} \gamma^{i}\right)=\bigoplus_{i=0}^{n} \widetilde{w} \widetilde{b}_{i} \gamma^{i}=\bigoplus_{i=0}^{n} \widetilde{y}_{i} \gamma^{i} \text { dengan } \widetilde{y}_{i}=\widetilde{w} \widetilde{b}_{i}
\end{aligned}
$$

Disini, $\widetilde{v} \widetilde{a}_{i}$ merupakan himpunan fuzzy yang $\alpha$-cutnya adalah interval $\left[\underline{a_{\alpha}} \otimes \underline{v_{\alpha}}\right.$, $\left.\overline{a_{\alpha}} \otimes \overline{v_{\alpha}}\right]$ untuk setiap $\alpha \in[0,1]$ dan $\left[\underline{a_{\alpha}} \otimes \underline{v_{\alpha}}, \overline{a_{\alpha}} \otimes \overline{v_{\alpha}}\right]=\left[\underline{a_{\alpha}}, \overline{a_{\alpha}}\right] \otimes\left[\underline{v_{\alpha}}, \overline{v_{\alpha}}\right]$. Karena $\widetilde{a}_{i}=\widetilde{b}_{i}$, maka $\alpha$-cutnya, sama yakni $\widetilde{a}_{i \alpha}=\widetilde{b}_{i \alpha}$. Dari sini diperoleh $\left[\underline{v_{\alpha}}, \overline{v_{\alpha}}\right.$ ]$=\left[\underline{w_{\alpha}}, \overline{w_{\alpha}}\right]$. Akibatnya $\underline{v_{\alpha}}=\underline{w_{\alpha}}$ dan $\overline{v_{\alpha}}=\overline{w_{\alpha}}$. Kemudian,

$$
\begin{aligned}
\tilde{x}_{i}=\widetilde{v} \widetilde{a}_{i} & =\left[\underline{a_{\alpha}} \otimes \underline{v_{\alpha}}, \overline{a_{\alpha}} \otimes \overline{v_{\alpha}}\right]=\left[\underline{a_{\alpha}}, \overline{a_{\alpha}}\right] \otimes\left[\underline{v_{\alpha}}, \overline{v_{\alpha}}\right] \\
& =\left[\underline{b_{\alpha}}, \overline{b_{\alpha}}\right] \otimes\left[\underline{w_{\alpha}}, \overline{w_{\alpha}}\right]=\left[\underline{b_{\alpha}} \otimes \underline{w_{\alpha}}, \overline{b_{\alpha}} \otimes \overline{w_{\alpha}}\right]=\widetilde{w} \widetilde{b}_{i}=\widetilde{y}_{i}
\end{aligned}
$$

sehingga diperoleh $\widetilde{v} p=\widetilde{w} p^{\prime}$. Dengan demikian, operasi eksternal yang didefinisikan pada $\Re_{\max }[\gamma]$ merupakan operasi yang terdefinisi dengan baik.

Untuk selanjutnya akan diselidiki sifat-sifat yang berlaku pada operasi $\oplus$ . Misalkan, untuk $p, q, r \in \mathcal{R}_{\max }[\gamma], p=\bigoplus_{i=0}^{n} \widetilde{a}_{i} \gamma^{i}$ dengan $\widetilde{a}_{i} \in \mathcal{R}_{\max }, q=$ $\bigoplus_{i=0}^{m} \widetilde{b}_{i} \gamma^{i}$ dengan $\widetilde{b}_{i} \in \mathcal{R}_{\max }$ dan $\quad r=\bigoplus_{i=0}^{k} \tilde{c}_{i} \gamma^{i}$ dengan $\tilde{c}_{i} \in \mathcal{R}_{\max }$. Tanpa mengurangi keumuman, diambil $n \geq m \geq k$, sehingga

$$
\begin{aligned}
{[p \oplus q] \oplus r } & =\left[\left(\bigoplus_{i=0}^{n} \tilde{a}_{i} \gamma^{i}\right) \oplus\left(\bigoplus_{i=0}^{m} \widetilde{b}_{i} \gamma^{i}\right)\right] \oplus\left(\bigoplus_{i=0}^{k} \tilde{c}_{i} \gamma^{i}\right) \\
& =\left[\left(\bigoplus_{i=0}^{n}\left(\widetilde{a}_{i} \widetilde{\oplus} \widetilde{b}_{i}\right) \gamma^{i}\right)\right] \oplus\left(\bigoplus_{i=0}^{k} c_{i} \gamma^{i}\right) \\
& \left.=\bigoplus_{i=0}^{n}\left[\left(\widetilde{a}_{i} \widetilde{\oplus} b_{i}\right) \widetilde{\oplus} \tilde{c}_{i}\right] \gamma^{i}\right)=\bigoplus_{i=0}^{n}\left[\widetilde{a}_{i} \widetilde{\oplus}\left(\widetilde{b}_{i} \widetilde{\oplus} \tilde{c}_{i}\right)\right] \gamma^{i} \\
& =\left(\bigoplus_{i=0}^{n} \tilde{a}_{i} \gamma^{i}\right) \oplus\left[\left(\bigoplus_{i=0}^{m}\left(\widetilde{b}_{i} \widetilde{\oplus} \tilde{c}_{i}\right) \gamma^{i}\right)\right]
\end{aligned}
$$




$$
\begin{aligned}
& =\left(\bigoplus_{i=0}^{n} \tilde{a}_{i} \gamma^{i}\right) \oplus\left[\left(\bigoplus_{i=0}^{m} \widetilde{b}_{i} \gamma^{i}\right) \oplus\left(\bigoplus_{i=0}^{k} \tilde{c}_{i} \gamma^{i}\right)\right] \\
& =p \oplus[q \oplus r]
\end{aligned}
$$

Dengan demikian, operasi $\oplus$ bersifat assosiatif pada $\mathcal{R}_{\max }[\gamma]$.

Berikutnya akan diselidiki sifat komutatif operasi $\oplus$ pada $\mathcal{R}_{\max }[\gamma]$. Untuk setiap $p, q \in \mathscr{R}_{\max }[\gamma]$ berlaku

$$
\begin{aligned}
p \oplus q & =\left(\bigoplus_{i=0}^{n} \widetilde{a}_{i} \gamma^{i}\right) \oplus\left(\bigoplus_{i=0}^{m} \widetilde{b}_{i} \gamma^{i}\right)=\bigoplus_{i=0}^{n}\left(\widetilde{a}_{i} \widetilde{\oplus} \widetilde{b}_{i}\right) \gamma^{i} \\
& =\bigoplus_{i=0}^{n}\left(\widetilde{b}_{i} \widetilde{\oplus} \widetilde{a}_{i}\right) \gamma^{i}=\left(\bigoplus_{i=0}^{m} \widetilde{b}_{i} \gamma^{i}\right) \oplus\left(\bigoplus_{i=0}^{n} \widetilde{a}_{i} \gamma^{i}\right)=q \oplus p
\end{aligned}
$$

Dengan demikian, operasi $\oplus$ bersifat komutatif pada $\mathcal{R}_{\max }[\gamma]$.

Polinomial nol $\varepsilon$ merupakan elemen pada $\Re_{\max }[\gamma]$, karena $\varepsilon=\bigoplus_{i=0}^{n} \widetilde{a}_{i} \gamma^{i}$, dengan $\widetilde{a}_{i}=\varepsilon$ adalah himpunan fuzzy dengan $\alpha$-cutnya adalah interval $[\varepsilon, \varepsilon]$. Untuk setiap $p=\bigoplus_{i=0}^{n} \widetilde{a}_{i} \gamma^{i} \in \mathcal{R}_{\max }[\gamma]$ berlaku

$$
p \oplus \varepsilon=\left(\bigoplus_{i=0}^{n} \quad \widetilde{a}_{i} \gamma^{i}\right) \oplus\left(\bigoplus_{m=0}^{n} \quad \tilde{\varepsilon} \gamma^{i}\right)=\bigoplus_{i=0}^{n}\left(\widetilde{a}_{i} \widetilde{\oplus} \tilde{\varepsilon}\right) \gamma^{i}=\bigoplus_{i=0}^{n} \quad \widetilde{a}_{i} \gamma^{i}=p
$$

Secara analog, juga berlaku $\varepsilon \oplus p=p$. Dengan demikian, $\mathcal{R}_{\max }[\gamma]$ mempunyai elemen nol yaitu polinomial nol $\mathcal{\varepsilon}$.

Untuk selanjutnya akan diselidiki sifat yang yang berlaku pada operasi pergandaan skalar. Untuk setiap $\widetilde{v}, \widetilde{w} \in \mathcal{R}_{\max }$ dan $p, q \in \mathcal{R}_{\max }[\gamma]$

i. $\widetilde{v}[p \oplus q] \quad=\widetilde{v}\left[\left(\bigoplus_{i=0}^{n} \widetilde{a}_{i} \gamma^{i}\right) \oplus\left(\bigoplus_{i=0}^{m} \widetilde{b}_{i} \gamma^{i}\right)\right]=\widetilde{v}\left[\bigoplus_{i=0}^{n}\left(\widetilde{a}_{i} \widetilde{\oplus} \widetilde{b}_{i}\right) \gamma^{i}\right]$

$$
\begin{aligned}
& =\bigoplus_{i=0}^{n} \widetilde{v}\left(\widetilde{a}_{i} \widetilde{\oplus} \widetilde{b}_{i}\right) \gamma^{i}=\bigoplus_{i=0}^{n}\left(\widetilde{v} \widetilde{a}_{i} \widetilde{\oplus} \widetilde{v} \widetilde{b}_{i}\right) \gamma^{i} \\
& =\left(\bigoplus_{i=0}^{n} \widetilde{v} \widetilde{a}_{i} \gamma^{i}\right) \oplus \quad\left(\bigoplus_{i=0}^{m} \widetilde{v} \widetilde{b}_{i} \gamma^{i} \quad\right)=\widetilde{v}\left(\bigoplus_{i=0}^{n} \widetilde{a}_{i} \gamma^{i}\right) \oplus
\end{aligned}
$$

$\widetilde{v}\left(\bigoplus_{i=0}^{m} \widetilde{b}_{i} \gamma^{i}\right)$

$$
=\widetilde{v} p \oplus \widetilde{v} q
$$


ii. $[\widetilde{v} \widetilde{\oplus} \widetilde{w}] p=[\widetilde{v} \widetilde{\oplus} \widetilde{w}]\left(\bigoplus_{i=0}^{n} \widetilde{a}_{i} \gamma^{i}\right)=\left(\bigoplus_{i=0}^{n}[\widetilde{v} \widetilde{\oplus} \widetilde{w}] \widetilde{a}_{i} \gamma^{i}\right)$

$$
\begin{aligned}
& =\left(\bigoplus_{i=0}^{n}\left[\widetilde{v} \widetilde{a}_{i} \widetilde{\oplus} \widetilde{w} \widetilde{a}_{i}\right] \gamma^{i}\right)=\left(\bigoplus_{i=0}^{n} \widetilde{v} \widetilde{a}_{i} \gamma^{i}\right) \oplus\left(\bigoplus_{i=0}^{n} \widetilde{w} \widetilde{a} \sim \gamma^{i}\right) \\
& =\widetilde{v}\left(\bigoplus_{i=0}^{n} \widetilde{a}_{i} \gamma^{i}\right) \oplus \widetilde{w}\left(\bigoplus_{i=0}^{n} \quad \widetilde{a}_{i} \gamma^{i}\right)=\widetilde{v} p \bigoplus \tilde{v} p
\end{aligned}
$$

iii. $\widetilde{v}[\widetilde{w} p]=\widetilde{v}\left[\widetilde{w}\left(\bigoplus_{i=0}^{n} \widetilde{a}_{i} \gamma^{i}\right)\right]=\widetilde{v}\left[\bigoplus_{i=0}^{n}\left(\widetilde{w} \widetilde{a}_{i}\right) \gamma^{i}\right]=\left[\bigoplus_{i=0}^{n} \widetilde{v}\left(\widetilde{w} \widetilde{a}_{i}\right) \gamma^{i}\right]$

$$
=\left[\bigoplus_{i=0}^{n}(\widetilde{v} \widetilde{w}) \widetilde{a}_{i} \gamma^{i}\right]=(\tilde{v} \widetilde{w})\left[\bigoplus_{i=0}^{n} \widetilde{a}_{i} \gamma^{i}\right]=(\tilde{v} \widetilde{w}) p
$$

Selanjutnya $\tilde{e} p=\tilde{e}\left(\bigoplus_{i=0}^{n} \quad \widetilde{a}_{i} \gamma^{i}\right)=\bigoplus_{i=0}^{n} \tilde{e} \widetilde{a}_{i} \gamma^{i}=\bigoplus_{i=0}^{n} \quad \tilde{a}_{i} \gamma^{i}=p$ dengan $\tilde{e}$ adalah elemen satuan pada $\mathfrak{R}_{\max }$, yakni himpunan fuzzy dengan $\alpha$-cutnya adalah interval $[e, e]$. Kemudian $\tilde{\varepsilon} p=\tilde{\varepsilon}\left(\bigoplus_{i=0}^{n} \widetilde{a}_{i} \gamma^{i}\right)=\bigoplus_{i=0}^{n} \quad \tilde{\varepsilon} \widetilde{a}_{i} \gamma^{i}=\bigoplus_{i=0}^{n} \tilde{\varepsilon} \gamma^{i}=\tilde{\varepsilon}$.

Dari uraian sebelumnya, terbukti bahwa operasi internal $\oplus$ pada $\mathcal{R}_{\max }[\gamma]$ dan operasi eksternal pergandaan skalar yang dikerjakan pada $\mathcal{R}_{\max }[\gamma]$ dan $\mathcal{R}_{\max }$ memenuhi $\oplus$ assosiatif , komutatif dan untuk setiap $\widetilde{v}, \widetilde{w} \in \mathcal{R}_{\max }$, dan $p, q \in$ $\mathcal{R}_{\max }[\gamma]$ berlaku $\widetilde{v}(p \oplus q)=\widetilde{v} p \oplus \widetilde{v} q,(\widetilde{v} \oplus \widetilde{\mathrm{w}}) p=\widetilde{v} p \oplus \widetilde{\mathrm{w}} p, \widetilde{v}(\widetilde{w} p)=(\widetilde{v} \widetilde{\mathrm{w}}) p$ , $\tilde{e} p=p$, serta $\tilde{\varepsilon} p=\varepsilon$. Dengan demikian, memenuhi aksioma-aksioma pada semi modul atas semi ring. Jadi, $\mathcal{R}_{\max }[\gamma]$ merupakan semimodul atas $\mathcal{R}_{\max }$. Dengan kata lain, himpunan polinomial fuzzy merupakan semi modul atas semi ring aljabar max-plus fuzzy.

\section{KESIMPULAN}

Operasi internal pada himpunan polinomial fuzzy dan operasi eksternal pada himpunan polinomial fuzzy atas aljabar max-plus fuzzy memenuhi aksiomaaksioma pada semi modul atas semi ring. Dengan demikian, himpunan polinomial fuzzy merupakan semi modul atas semi ring aljabar max-plus fuzzy. 


\section{DAFTAR PUSTAKA}

Bacelli, F, et al. 2001. Synchronization and Linearity. New York : John Wiley \& Sons.

Rudhito, A, 2007. Semimodul Bilangan Fuzzy atas Aljabar Max-Plus Bilangan Fuzzy. Prosiding Seminar Nasional Matematika, F MIPA UPI\&IndoMS 2007

----------- 2006. Aljabar Max-Plus Bilangan Kabur. Artikel Berkala MIPA

Susilo, F. 2006. Himpunan dan Logika Kabur serta Aplikasinya. Yogyakarta : Graha Ilmu

Zimmermann, H.J. 1991. Fuzzy Set Theory and Its Applications. Kluwer Academic Publishers, USA 
